\title{
Metalurgia e materiais \\ Análise de silício metálico em escória por deslocamento de coluna de mercúrio
}

\author{
Analysis of the silicon metal obtained from \\ slag by displacement of a mercury column
}

\author{
Aline Cristina P. Sousa de Caux \\ Mestre em Engenharia Industrial, \\ Vamtec S/A. \\ acaux@vamtecsa.com.br
}

Fernanda Gonçalves Nascimento Graduanda em Engenharia Ambiental, Unileste-MG.

fernandag.nascimento@yahoo.com.br

\section{Márcio Farias Silveira \\ Engenheiro Ambiental, Unileste-MG. marcio.silveira1@yahoo.com.br}

Pedro José Nolasco Sobrinho Pós-Doutor em Engenharia Metalúrgica, Vamtec S/A.

nolasco@vamtecsa.com.br

\section{Resumo}

Nesse trabalho, é apresentada uma proposta para a análise de silício metálico em escória da produção de ferro-silício, através da quantificação do hidrogênio liberado pela reação da amostra com hidróxido de sódio, mensurado através do deslocamento de coluna de mercúrio acoplada a sistema fechado. $\mathrm{O}$ valor obtido é comparado ao deslocamento provocado por amostra-padrão. O método foi comparado à metodologia gravimétrica tradicionalmente utilizada, que se baseia na mesma reação, entretanto o $\mathrm{Si}^{\circ}$ é quantificado através da conversão ácida do silicato formado na reação em sílica. Os resultados mostraram que o método proposto possui elevada correlação com a metodologia gravimétrica, com custo analítico inferior e maior rapidez de execução. Foram observados os valores de 0,177\%, para o Limite de Detecção, e de 0,574\%, para o Limite de Quantificação da metodologia proposta, ambos satisfatórios para as concentrações que esse trabalho procura analisar.

Palavras-chave: Silício, limite de detecção e limite de quantificação.

\begin{abstract}
This work presents a silicon metal analysis proposal for the slag from IronSilicon production. It quantifies the hydrogen liberated by the sample's reaction with sodium hydroxide and reads the mercury column displacement in a closed system. The obtained value is compared with the displacement of a reference sample. The proposed method was compared with a traditional gravimetric method that is based on the same method, but where sodium hidroxide is quantified when the silicate acid formed in the reaction is transformed into silica. The results showed that the proposed method had a high correlation with the gravimetric method, but at alower cost and quicker performance. A Detection Limit of $0.177 \%$ and a Quantification Limit of $0.574 \%$ were observed, of which both were satisfactory for this aim.
\end{abstract}

Keywords: Silicon, detection limit, quantification limit.

\section{Introdução}

A busca do desenvolvimento de técnicas de reutilização de resíduos na criação de produtos de grande valor agregado vem ao encontro de uma tendência mundial de preservação ambiental (Caux et al., 2007). Pesquisas visando, agregar valor e qualidade aos resíduos sólidos industriais e urbanos têm ganhado espaço em muitos laboratórios e instituições com trabalhos que demonstram o grande potencial do investimento na tecnologia de seu reaproveitamento (Yalçin \& Sevinç, 2000; Gemelli et al., 2001; Ferraris et al., 2001; 
Leite \& Molin, 2002; Méndez et al., 2003; Jonker \& Potgieter, 2005). Esses desenvolvimentos devem ser sustentados pela caracterização analítica confiável e rápida, assegurando, assim, resultados precisos com a agilidade exigida pelos ambientes industriais, além do baixo custo analítico.

O silício ocorre na natureza na forma combinada, é encontrado em, praticamente, todas as rochas, areias e solos. Entre seus compostos naturais, alguns dos mais importantes são: quartzo $-\mathrm{SiO}_{2}$, asbestos - $\mathrm{H}_{4} \mathrm{Mg}_{3} \mathrm{Si}_{2} \mathrm{O}_{9}$, zeolita - $\mathrm{Na}_{2}\left(\mathrm{Al}_{2} \mathrm{Si}_{3} \mathrm{O}_{10}\right) \cdot \mathrm{H}_{2} \mathrm{O}$ e mica $\mathrm{K}_{2} \mathrm{Al}_{2}\left(\mathrm{Al}_{2} \mathrm{Si}_{3} \mathrm{O}_{10}\right) \cdot \mathrm{H}_{2} \mathrm{O}$ (Peixoto, 2001). Sua produção em escala industrial acontece pela redução carbotérmica do quartzo em fornos de arco elétrico, onde a sílica $\left(\mathrm{SiO}_{2}\right)$ é reduzida com o uso de eletrodos de carbono em temperaturas superiores a $1900{ }^{\circ} \mathrm{C}$ (Suzuki \& Pereira, 2003; Mei, 2008). Os resíduos gerados

Comumente o silicato de sódio formado é transformado em sílica por trata-

A metodologia de análise, para a determinação do teor de silício metálico, proposta nesse trabalho baseia-se na Reação 1 descrita anteriormente, entretanto o composto quantificado será o hidrogênio liberado na reação, que deslocará uma coluna de mercúrio de maneira análoga a determinação de alumínio metálico des-

\section{Materiais e métodos}

\section{Materiais}

Foi utilizado o Padrão IPT 135 - Silício Metálico Grau Químico com pureza de $99,56 \%$ de silício metálico fornecido pelo Instituto de Pesquisas Tecnológicas - IPT. O Padrão foi diluído conforme os

\section{Métodos}

Metodologia de rotina: Ataque da amostra com hidróxido de sódio, formando silicato de sódio e liberando hidrogênio. A amostra deve ficar em repouso até decantação dos demais constituintes (entre eles a sílica). Em seguida, é retirada alíquota (do sobrenadante, silicato de sódio), que recebe tratamento ácido (ácidos perclórico e clorídrico), formando sílica, que é quan- na fabricação de silício metálico e ligas de $\mathrm{Fe}-\mathrm{Si}$, como as poeiras e as escórias, após a caracterização química e física, podem ser transformados em produtos auxiliares para a fabricação de ligas e aço, mais especificamente escórias sintéticas para o ajuste da composição química do gusa ou desoxidantes.

No processo de fabricação de aço, o ajuste do teor de silício do gusa pode ser realizado através de adições de fontes de silício metálico. É de suma importância a determinação do teor de silício metálico do produto, para se evitarem erros nos cálculos de balanço de massa e térmico do processo em questão, pois é na forma metálica que ocorrerá a maior contribuição para incorporação desse elemento no metal e/ou desoxidação da escória e/ ou do metal. O silício na forma de sílica $\left(\mathrm{SiO}_{2}\right)$, por exemplo, permanecerá na escória do processo e somente contribuirá para o aumento do silício total do produ-

$\mathrm{Si}+2 \mathrm{NaOH}+\mathrm{H}_{2} \mathrm{O} \rightarrow \mathrm{Na}_{2} \mathrm{SiO}_{3}+2 \mathrm{H}_{2}$

mento ácido. A sílica formada é filtrada, calcinada e pesada.

$$
\mathrm{SiO}_{3}^{-2}+2 \mathrm{H}^{+} \rightarrow \mathrm{SiO}_{2}+\mathrm{H}_{2} \mathrm{O}
$$

crito na NBR 8959 (Materiais Exotérmicos para Lingotamento de Aço - Determinação de Alumínio Metálico). O volume de hidrogênio liberado será comparado ao volume liberado por uma amostrapadrão (com teor de silício conhecido) e desta forma, o silício metálico poderá ser quantificado de maneira indireta. to e do volume de escória, forçando, muitas vezes, o aciarista a realizar adições de outros materiais e fundentes para o ajuste da basicidade do processo, visando ao seu controle (Rizzo, 2005; Rizzo, 2006a; Rizzo, 2006b). Por isso, a determinação rápida e precisa do teor de silício metálico de produtos auxiliares utilizados na metalurgia e siderurgia, na fabricação de ligas metálicas, gusa e aço, independentemente do processo, toma um caráter de vital importância.

Para quantificar o silício metálico e a sílica presentes, simultaneamente, numa amostra é comum utilizar-se a Difração de Raios X (Gazulla et al., 2006). Nem sempre os laboratórios dispõem desse equipamento e uma das metodologias alternativas utilizadas, para a análise de silício metálico em escória consiste na sua reação com hidróxido de sódio, que tem, como produtos, o silicato de sódio e o hidrogênio, conforme a reação (Miller, 2009):

(Reação 1)

(Reação 2)

Para validação da metodologia, utilizou-se padrão certificado IPT 135. Foram realizadas análises comparativas pelas duas metodologias.

Foi realizado, ainda, o cálculo do Limite de Detecção e Limite de Quantificação da metodologia proposta (NBR ISO/IEC 17025). teores de 1,$0 ; 5,0 ; 7,5 ; 10$ e 12,5\% em massa com areia quartzoza certificada, IPT 61, para avaliação do Limite de Detecção e Limite de Quantificação.

Para comparação das metodolo- gias, utilizou-se amostra de escória, que foi analisada pelos dois procedimentos com dez repetições. Em complemento, foram analisadas 35 amostras de escória de silício escolhidas aleatoriamente. tificada após filtração e calcinação. Essa metodologia demanda grande tempo de análise, devido ao repouso e apresenta o risco de que parte da sílica presente no precipitado possa compor a alíquota para determinação do Si, fazendo com que o valor obtido seja maior que o verdadeiro.

Metodologia proposta: Reação da amostra com hidróxido de sódio em sistema fechado conforme descrito na Figura 1. O hidrogênio liberado é quantificado através do deslocamento de coluna de mercúrio e comparado ao valor obtido pelo deslocamento provocado por amostra-padrão.

A preparação das amostras de escória consistiu na homogeneização seguida de quarteamento, secagem e pulveriza- 
Figura 1

Esquema do aparato utilizado para análise de silício metálico.

ção em moinho-panela.

Para a obtenção dos teores de $\mathrm{Si}^{\circ}$ desejados, o Padrão IPT 135 foi diluído com a areia quartzoza certificada, IPT 61. Após cálculos das massas necessárias dos materiais para cada concentração de interesse $(1,0 ; 5,0 ; 7,5 ; 10$ e $12,5 \%$ em

\section{Resultados}

Para análise do teor de silício metálico, é necessário calcular, previamente, o fator de correção utilizando amostrapadrão. Através do conhecimento do des-

Onde:

$\% \mathrm{Si}=$ Teor de silício do padrão IPT 135 $-99,56 \%$

$\mathrm{m}=$ massa de padrão utilizada, $\mathrm{g}$

Onde:

$\mathrm{L}=$ Leitura do deslocamento provocado na coluna de mercúrio, $\mathrm{mm}$;

$\mathrm{FC}=$ Fator de Correção obtido anteriormente, \%.g.mm ${ }^{-1}$;

$\mathrm{m}=$ massa de amostra, $\mathrm{g}$.

A Figura 2 mostra a correlação entre

Figura 2

Curva Padrão versus Método Proposto.

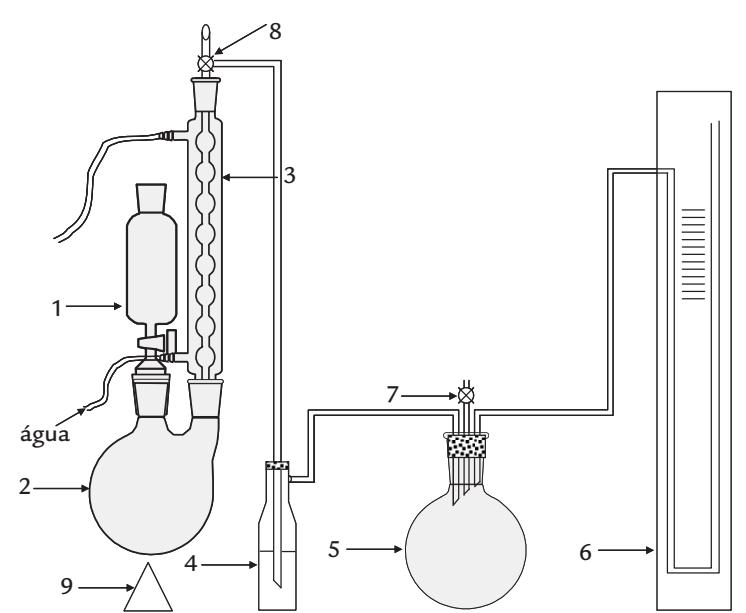

1. Funil ou bureta comum ou graduado acoplável ao balão de reação

2. Balão de reação de $500 \mathrm{~mL}$

3. Condensador

4. Frasco de lavagem de gases contendo $150 \mathrm{~mL}$ de solução de ácido sulfúrico $10 \%$

5. Balão de $3000 \mathrm{~mL}$

6. Coluna de mercúrio com escala em milímetros (resolução de $1 \mathrm{~mm}$ )

7. Controle interno de pressão

8. Controle de vácuo

9. Manta aquecedora

massa), pesou-se o Padrão IPT 135 e o Padrão IPT 61, em balança analítica de precisão, modelo AG200, Gehaka, e, em seguida, realizou-se homogeneização até mistura completa.

Os resultados obtidos foram avaliados estatisticamente via Microsoft Excel:
- Comparação entre resultados: Teste $\mathrm{t}$ (Box et al., 1978; Montgomery \& Runger, 2003), gráfico de dispersão.

- Limite de Detecção e Limite de Quantificação: Regressão linear (Box et al., 1978). locamento da coluna de mercúrio para uma amostra com teor de silício metálico conhecido, é possível correlacionar ao deslocamento provocado por amostras com

$$
\mathrm{FC}=\frac{\% \mathrm{Si} \times \mathrm{m}}{\mathrm{L}}
$$

$\mathrm{L}=$ leitura do deslocamento provocado na coluna de mercúrio, $\mathrm{mm}$

Em seguida realizaram-se análises dos padrões obtidos por diluição e

$$
\% \mathrm{Si}=\frac{\mathrm{LxFC}}{\mathrm{m}}
$$

as concentrações de $\mathrm{Si}^{\circ}$ nos padrões (obtidas através das diluições do IPT 135) no eixo das abscissas e os valores obtidos pelo método proposto no eixo das ordenadas.

A Tabela 1 mostra os resultados do Teste $\mathrm{t}$, com 95\% de confiança para as análises realizadas na amostra de escória teores desconhecidos e calcular as concentrações de silício metálico. Foi utilizado o Padrão IPT 135 puro para determinação do Fator de Correção, através da equação:

(Equação 1)

correlacionaram-se os valores teóricos com os valores obtidos pela análise proposta. O cálculo dos resultados foi feito utilizando-se a equação:

(Equação 2)

pelas duas metodologias.

A Figura 3 mostra a correlação entre os resultados obtidos pelo método de rotina e pela metodologia proposta, demonstrando excelente correlação entre os dois procedimentos para determinação de $\mathrm{Si}^{\circ}$ em 35 amostras de escória.

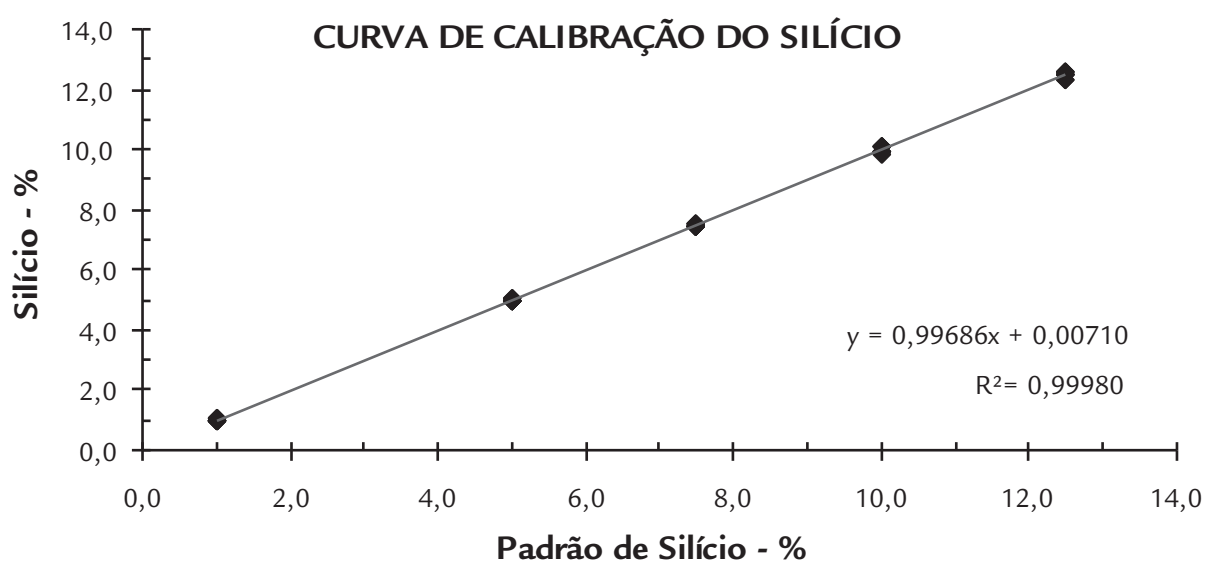




\section{Discussão}

Através da análise da Figura 2, pode-se observar excelente aproximação ( $\mathrm{R}$ 1.0) do modelo linear $(\mathrm{y}=\mathrm{ax}+\mathrm{b})$ com intercepto $b$ tendendo a zero e inclinação a tendendo a 1 , levando o modelo a simplificação $\mathrm{y}=\mathrm{x}$, ou seja, os valores observados são iguais ao padrão. O Desviopadrão dos Resíduos (RSD - Residues Standard Deviation) foi obtido através

O Limite de quantificação (LQ) de um método analítico é a menor concentração do analito que pode ser determi-

da comparação entre as concentrações reais obtidas em análise e as concentrações calculadas, obtendo-se o valor de 0,05669 (Anexo 1), que foi utilizado nos cálculos do Limite de Detecção (LD) e do Limite de Quantificação (LQ).

O LD é definido como a menor concentração do analito em uma amostra, que pode ser detectada, mas não

$$
\mathrm{LD}=3 * \mathrm{RSD}+0,0071
$$

nada com precisão e exatidão, aceitáveis, sob determinadas condições experimentais (Barros, 2002). O LQ encontrado,

$$
\mathrm{LQ}=10 * \mathrm{RSD}+0,0071
$$

\begin{tabular}{c|c|c}
\cline { 2 - 3 } & Método Rotina & Método Proposto \\
\hline Média & 22,664 & 22,285 \\
\hline Variância & 0,309982222 & 0,246316667 \\
\hline Observações & 10 & 10 \\
\hline Correlação de Pearson & 0,113635586 & \\
\hline Hipótese da diferença de média & 0 & \\
\hline Gl & 9 & \\
\hline Stat $t$ & 1,706067148 & \\
\hline $\mathrm{P}(\mathrm{T}<=\mathrm{t})$ uni-caudal & 0,061091359 & \\
\hline $\mathrm{t}$ crítico uni-caudal & 1,833112923 & \\
\hline $\mathrm{P}(\mathrm{T}<=\mathrm{t})$ bi-caudal & 0,122182717 & \\
\hline $\mathrm{t}$ crítico bi-caudal & 2,262157158 & \\
\hline
\end{tabular}

Para qualquer método analítico, é importante avaliar a concentração mínima na qual o método pode ser aplicado. O LD é a menor concentração que poderá ser detectada pelo método proposto e o LQ é um parâmetro que marca a habilidade de um processo de medição de quantificar, adequadamente, o elemento ou substância de interesse (Souza, 2007). Os resultados de LD e LQ encontrados, para a metodologia proposta, mostram níveis aceitáveis para os valores que esse trabalho deseja analisar, que são, geralmente, superiores a $10 \%$ de $\mathrm{Si}^{\circ}$, mais al-

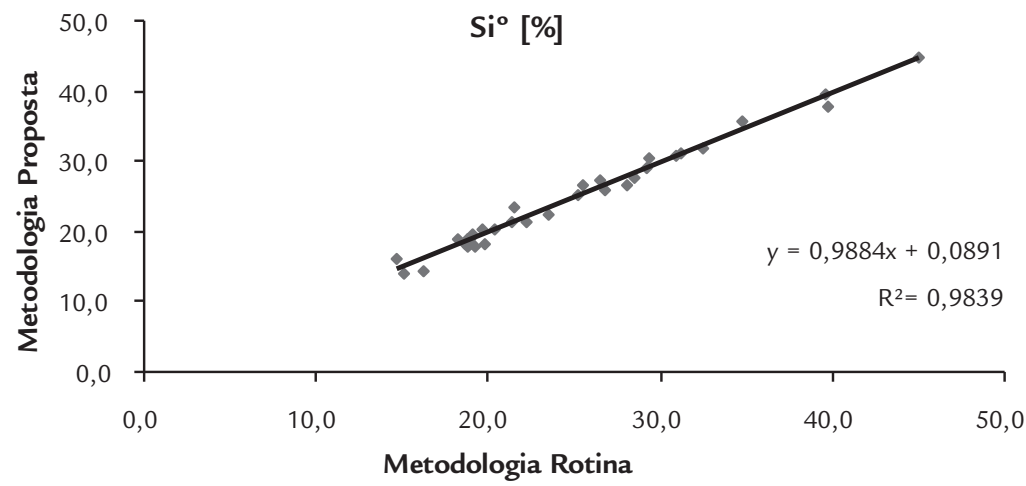

necessariamente quantificada, sob determinadas condições experimentais. Normalmente é determinado apenas para esclarecer o limite inferior da faixa de operação prática do método (Eurachem, 1998). O LD encontrado, para o método proposto, foi de $0,177 \%$ calculado de acordo com a Equação 3 (Oliveira, 2007):

(Equação 3)

para o método proposto, foi de $0,574 \%$ calculado de acordo com a Equação 4 (Oliveira, 2007):

(Equação 4)

Tabela 1

Teste t: Método Rotina versus Método Proposto.

rotina. A redução da quantidade de reagentes bem como do número de tipos de reagentes utilizados devem ser também levados em consideração devido ao ganho proporcionado no que diz respeito ao aspecto ambiental. $\mathrm{Na}$ metodologia proposta, utiliza-se, como reagente, apenas o hidróxido de sódio, eliminando a etapa de tratamento ácido realizada para conversão do silicato de sódio em sílica na metodologia gravimétrica. Outro ponto relevante é o tempo de análise, que foi reduzido de 12 horas para, aproximadamente, 30 minutos.

Figura 3

Método Proposto versus Método Rotina. 
Anexo 1

Resultados de silício metálico, LD e LQ.

\begin{tabular}{|c|c|c|c|}
\hline $\begin{array}{c}\text { Valor real do padrão, } \\
\% \text { em massa }\end{array}$ & $\begin{array}{l}\text { Resultado da Análise - } \\
\% \text { em massa }\end{array}$ & $\begin{array}{l}\text { Concentração - \% em massa } \\
\text { (REGRESSÃO), XCalReg }\end{array}$ & $\begin{array}{c}\text { (XCalReg - } \\
\text { XReal) })^{2}\end{array}$ \\
\hline 1 & 1,00 & 0,9960 & $1,6 \mathrm{E}-05$ \\
\hline 1 & 1,01 & 1,0061 & 3,7E-05 \\
\hline 1 & 1,01 & 1,0061 & 3,7E-05 \\
\hline 1 & 1,01 & 1,0061 & 3,7E-05 \\
\hline 1 & 1,01 & 1,0061 & $3,7 \mathrm{E}-05$ \\
\hline 1 & 1,02 & 1,0161 & $2,6 \mathrm{E}-04$ \\
\hline 1 & 1,01 & 1,0061 & 3,7E-05 \\
\hline 1 & 1,00 & 0,9960 & $1,6 \mathrm{E}-05$ \\
\hline 1 & 1,02 & 1,0161 & $2,6 \mathrm{E}-04$ \\
\hline 1 & 1,01 & 1,0061 & 3,7E-05 \\
\hline 5 & 4,98 & 4,9886 & $1,3 \mathrm{E}-04$ \\
\hline 5 & 4,98 & 4,9886 & $1,3 \mathrm{E}-04$ \\
\hline 5 & 4,93 & 4,9384 & $3,8 \mathrm{E}-03$ \\
\hline 5 & 4,98 & 4,9886 & $1,3 \mathrm{E}-04$ \\
\hline 5 & 4,98 & 4,9886 & $1,3 \mathrm{E}-04$ \\
\hline 5 & 4,93 & 4,9384 & $3,8 \mathrm{E}-03$ \\
\hline 5 & 4,93 & 4,9384 & $3,8 \mathrm{E}-03$ \\
\hline 5 & 5,03 & 5,0387 & $1,5 \mathrm{E}-03$ \\
\hline 5 & 5,03 & 5,0387 & $1,5 \mathrm{E}-03$ \\
\hline 5 & 4,98 & 4,9886 & $1,3 \mathrm{E}-04$ \\
\hline 7,5 & 7,47 & 7,4864 & $1,8 \mathrm{E}-04$ \\
\hline 7,5 & 7,55 & 7,5667 & 4,4E-03 \\
\hline 7,5 & 7,47 & 7,4864 & $1,8 \mathrm{E}-04$ \\
\hline 7,5 & 7,55 & 7,5667 & 4,4E-03 \\
\hline 7,5 & 7,4 & 7,4162 & $7,0 \mathrm{E}-03$ \\
\hline 7,5 & 7,47 & 7,4864 & $1,8 \mathrm{E}-04$ \\
\hline 7,5 & 7,47 & 7,4864 & $1,8 \mathrm{E}-04$ \\
\hline 7,5 & 7,55 & 7,5667 & 4,4E-03 \\
\hline 7,5 & 7,47 & 7,4864 & $1,8 \mathrm{E}-04$ \\
\hline 7,5 & 7,47 & 7,4864 & $1,8 \mathrm{E}-04$ \\
\hline 10 & 9,96 & 9,9852 & $2,2 \mathrm{E}-04$ \\
\hline 10 & 10,06 & 10,0848 & $7,2 \mathrm{E}-03$ \\
\hline 10 & 10,06 & 10,0842 & $7,1 \mathrm{E}-03$ \\
\hline 10 & 9,96 & 9,9860 & $2,0 \mathrm{E}-04$ \\
\hline 10 & 9,86 & 9,8839 & $1,3 \mathrm{E}-02$ \\
\hline 10 & 9,96 & 9,9842 & $2,5 \mathrm{E}-04$ \\
\hline 10 & 9,96 & 9,9842 & $2,5 \mathrm{E}-04$ \\
\hline 10 & 10,06 & 10,0846 & 7,2E-03 \\
\hline 10 & 10,06 & 10,0846 & $7,2 \mathrm{E}-03$ \\
\hline 10 & 9,96 & 9,9842 & 2,5E-04 \\
\hline 12,5 & 12,32 & 12,3517 & $2,2 \mathrm{E}-02$ \\
\hline 12,5 & 12,45 & 12,4826 & $3,0 \mathrm{E}-04$ \\
\hline 12,5 & 12,45 & 12,4815 & $3,4 \mathrm{E}-04$ \\
\hline 12,5 & 12,45 & 12,4838 & $2,6 \mathrm{E}-04$ \\
\hline 12,5 & 12,45 & 12,4829 & $2,9 \mathrm{E}-04$ \\
\hline 12,5 & 12,57 & 12,6025 & $1,0 \mathrm{E}-02$ \\
\hline 12,5 & 12,45 & 12,4821 & $3,2 \mathrm{E}-04$ \\
\hline 12,5 & 12,32 & 12,3517 & $2,2 \mathrm{E}-02$ \\
\hline 12,5 & 12,57 & 12,6025 & $1,0 \mathrm{E}-02$ \\
\hline \multirow[t]{4}{*}{12,5} & 12,57 & 12,6025 & $1,0 \mathrm{E}-02$ \\
\hline & & RSD & 0,05669 \\
\hline & & LD, \% em massa & 0,177 \\
\hline & & LQ, \% em massa & 0,574 \\
\hline
\end{tabular}

\section{Conclusão}

O método proposto apresentou menor custo e maior rapidez de execução em relação ao método gravimétrico. Ambientalmente pode-se dizer que houve um ganho significativo com a redu-

\section{Agradecimentos}

Os autores agradecem à VAMTEC S/A pela oportunidade e apoio financeiro para realização deste trabalho e aos senho-

\section{Referências bibliográficas}

ção de quantidades e tipos de reagentes utilizados. A correlação entre os resultados obtidos nas duas metodologias foi de 98,39\%. O Limite de Detecção e o Limite de Quantificação encontrados foram de $0,177 \%$ e $0,574 \%$,respectivamente, e foram considerados satisfatórios para as concentrações que esse trabalho deseja analisar.

cução das análises químicas. res Dewison Fernandes, Douglas Marquioli Baião, Marcelo Rangel de Souza Santos e Wilien Dias pelo esmero na exe- 
térmicos Para Lingotamento de Aço - Determinação de Alumínio Metálico. Rio de Janeiro, 1985.

ASSOCIAÇÃO BRASILEIRA DE NORMAS TÉCNICAS. NBR ISO/IEC 17025: Requisitos gerais para competência de laboratórios de ensaio e calibração. Rio de Janeiro, 2005.

BARROS, C. B. Validação de Métodos Analíticos. Biológico, São Paulo, v.64, n.2, p.175177, jul./dez. 2002.

BOX, G. E. P et al. Statistics for experimenters. New York: John Wiley \& Sons, Inc, 1978. $653 \mathrm{p}$.

CAUX, L. S. et al. Análise da viabilidade técnica da utilização de resíduos da indústria de celulose kraft na produção de corpos cerâmicos. In: CONGRESSO ANUAL DA ABM, 62. Anais...Vitória: ABM, 2007. p. 2215-2223.

EURACHEM: The fitness for purpose of analytical methods: a laboratory guide to method validation and related topics. 1998. Disponível em: <http://www.eurachem.org/ guides/valid.pdf>. Acesso em: 01 out. 2009.

FERRARIS, M. et al. Glass matrix composites from solids waste materials. Journal of The European Ceramic Society, Bologna, v. 21, n. 4, p. 453-460, Apr. 2001.

GAZULLA, M. F. et al. Physico-chemical characterisation of silicon carbide refractories. Journal of the European Ceramic Society, Bologna, v. 26, n.15, p. 3451-3458, 2006.

GEMELLI, E., CAMARGO, N. H. A., BRESCANSIN, J. Evaluation of Paper Industry Wastes in Construction Material Applications. Materials Research, São Carlos, v. 4, n. 4, p. 297-304, Oct. 2001.

JONKER, A., POTGIETER, J. H. An evaluation of selected waste resources for utilization in ceramic materials applications. Journal of The European Ceramic Society, Bologna, v. 25, n.13, p. 3145-3149, Aug. 2005.

LEITE, M. B., MOLIN, D. D. Avaliação da atividade pozolânica do material cerâmicos presente no agregado reciclado de resíduo de C\&D. Sitientibus, Feira de Santana, n. 26, p. 111-130, jan./jun. 2002.

MEI, P. R. Silício: graus de pureza e aplicação. Disponível em: < lffe.dema.fem.unicamp. googlepages.com/Silicio-Cetem-Artigoapresentado20-11.pdf>. Acesso em: 02 out. 2009.

MÉNDEZ, A. A. et al. Kinetic thermal analysis of glass ceramic from industrial wastes. Journal of Non-Crystalline Solids, v. 329, n. 1-3, p. 73-76, Nov. 2003.

MILLER, E. Silicon Literature Review and Reaction Data. Department of Chemistry San Juan College, New México. Disponível em: <http://www.sanjuancollege.edu/ documents/ MathSci/CHEM/Silicon\%20Literature\%20Review\%20and\%20Data. pdf>. Acesso em: 02 out. 2009.

MONTGOMERY, D. C., RUNGER, G.C. Estatística aplicada e probabilidade para engenheiros. Rio de Janeiro: LTC, 2003. 463 p.

OLIVEIRA, F. M. Aplicação e testes do método APHA-4500- $\mathrm{NO}_{3}-\mathrm{C}$ para determinação de nitratos em águas por leitura em UV com segunda-derivada. Revista Analytica, São Paulo, n. 27, p. 80-88, 2007.

PEIXOTO, E. M. A. Silício. Química Nova na Escola, São Paulo, n. 14, 2001. Disponível em: < http://qnesc.sbq.org.br/online/qnesc14/v14a12.pdf>. Acesso em: 03 out. 2009.

RIZZO, E. M. S. Introdução aos processos de preparação de matérias-primas para o refino do aço. São Paulo: Associação Brasileira de Metalurgia e Materiais, 2005. 69 p.

RIZZO, E. M. S. Introdução aos processos de refino primário dos aços nos fornos elétricos a arco. São Paulo: Associação Brasileira de Metalurgia e Materiais, 2006a. 102 p.

RIZZO, E. M. S. Introdução aos processos de refino secundário dos aços. São Paulo: Associação Brasileira de Metalurgia e Materiais, 2006b. 102 p.

SOUZA, S. V. C. Procedimento para validação intralaboratorial de métodos de ensaio: delineamento e aplicabilidade em análises de alimentos. Belo Horizonte: Faculdade de Farmácia da UFMG, 2007. 296 p.

SUZUKI, C. K., PEREIRA, J. T. V. Energia solar e produção de silício metálico baseado no programa QITS (“Quartz Industrial Trade System”). In: ENCONTRO DE ENERGIA NO MEIO RURAL, 3., 2000, Campinas. Disponível em: <http://www. proceedings.scielo.br>. Acesso em: 05 out. 2009.

YALÇIN, N., SEVINÇ, V. Utilization of bauxite waste in ceramic glazes. Ceramics International, v. 26, n.5, p. 485-493, 2000.

Artigo recebido em 17 de dezembro de 2009. Aprovado em 03 de maio de 2011.

318 REM: R. Esc. Minas, Ouro Preto, 64(3), 313-318, jul. set. | 2011 